\title{
Towards a 3D Space Radiation Transport Code
}

\author{
J.W. Wilson, R.K.Tripathi \\ NASA Langley Research Center, Hampton, VA \\ F.A. Cucinotta \\ NASA Johnson Space Center, Houston, TX \\ J.H. Heinbockel, J. Tweed \\ Old Dominion University, Norfolk, VA
}

Copyright $\odot 2002$ Society of Automotive Engineers, Inc.

\begin{abstract}
High-speed computational procedures for space radiation shielding have relied on asymptotic expansions in terms of the off-axis scatter and replacement of the general geometry problem by a collection of flat plates. This type of solution was derived for application to human rated systems in which the radius of the shielded volume is large compared to the off-axis diffusion limiting leakage at lateral boundaries. Over the decades these computational codes are relatively complete and lateral diffusion effects are now being added. The analysis for developing a practical full $3 \mathrm{D}$ space shielding code is presented.
\end{abstract}

\section{INTRODUCTION}

Early methods of space radiation shield evaluation relied largely on Monte Carlo codes [1,2] and made important contributions to NASA engineering programs. Yet, slow computational procedures did not allow progress in coupling to spacecraft geometry for over 30 years (compare Alsmiller et al. [3], Armstrong et al. [4]) and did not allow early entry of radiation constraints into the design process and off-optimum Monte Carlo solutions to shielding problems continue to plague final designs [5-7]. Simulations with full 3D Monte Carlo codes often use questionably simplified 1D shielding geometry models to increase computational speed in which shielding for an ISS module is approximated as a very long $20.7 \mathrm{~g} / \mathrm{cm}^{2}$ thick aluminum cylindrical shell [4] leading to an overestimate of the neutron flux within ISS since neutron leakage is underestimated in these homogenized configurations. The "3D calculations" used in current ISS Monte Cario studies are routinely exceeded in their correctness in coupling to spacecraft geometry by HZETRN [6-8]. In addition to spacecraft geometry simplifications, the astronauts in Monte Carto calculations are approximated by a $30-\mathrm{cm}$ sphere of tissue [3,4]. Conversely, the use of a 3D Monte Carlo code within such a simplified 1D geometry for ISS will lead to erroneous underestimates of the trapped charged particle exposure since anisotropic shield distributions within ISS have long been known to be a major factor (2 to 5) in astronaut organ exposure [9].

The reason for such simplifications using Monte Carlo methods is easy to understand. To map the interior of an ISS module requires at least $10^{6}$ events within each volume about a field point. With $10^{3}$ points for a field map, this requires $10^{9}$ events to map an interior field. Each particle track from boundary to exit will experience $10^{3}-10^{4}$ events requiring a ray trace per event leading to $10^{12}-10^{13}$ ray traces for a field evaluation. In our 16A ISS configuration in which only the HAB module is currently represented in detail [6], 14 seconds is required to evaluate $10^{3}$ rays with a 12 processor script on an Origin parallel machine or approximately $14 \mathrm{msec}$ per ray using optimized ray tracing methods [10]. The Monte Carlo interior field evaluation from ray tracing alone on a 12 processor Origin would require $10^{10}-10^{11}$ seconds. It should be clear that Monte Carto methods would not be useful in design optimization.

The development of high-speed deterministic procedures allows early entry of radiation constraints into the design process without simplification of the shield geometry [11] but Monte Carlo methods could still play a role in future final design evaluation with full geometry provided more efficient ray trace methods can be found, massively parallel machines are used, and/or a radically different approach to ray tracing can be found. Current Monte Carlo methods simply use trivial geometry, which is computationally efficient [4], but provide generally incorrect results and do not meet the requirement for efficient linkage to spacecraft geometry or biological models.

In the present report, we will give an overview of the salient features allowing the development of high- 
performance computational procedures for evaluation of the 3D geometry of spacecraft with astronauts. All of the steps in the process have been demonstrated at some minimal conceptual level and much of the software is available in terms of a computationally efficient first order approximation. Only the final assembly of the software package with the spacecraft/astronaut geometry and further evaluation of the cross section database is required. This last step will require a well-defined laboratory and flight validation of the program.

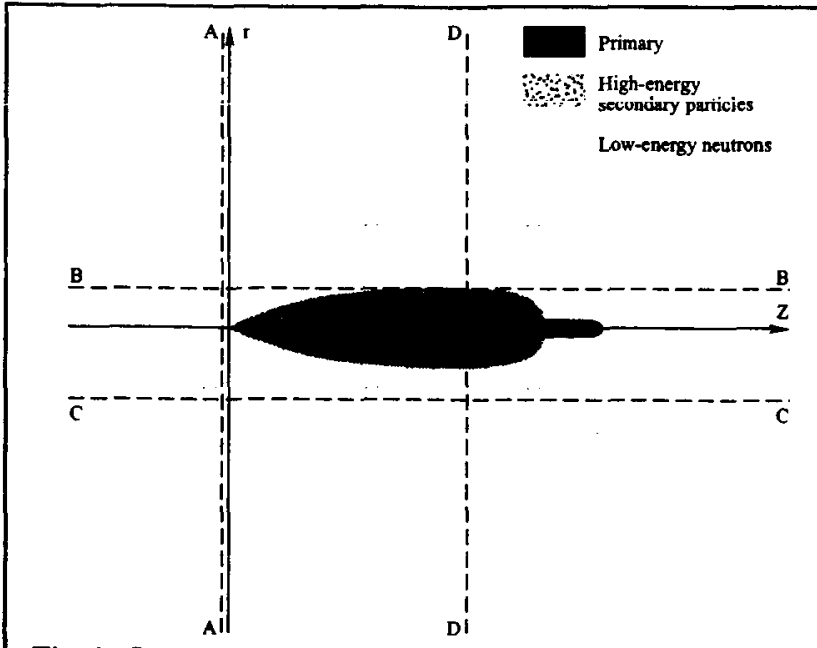

Fig.1. Schematic of a point source of monoenergetic partilcles

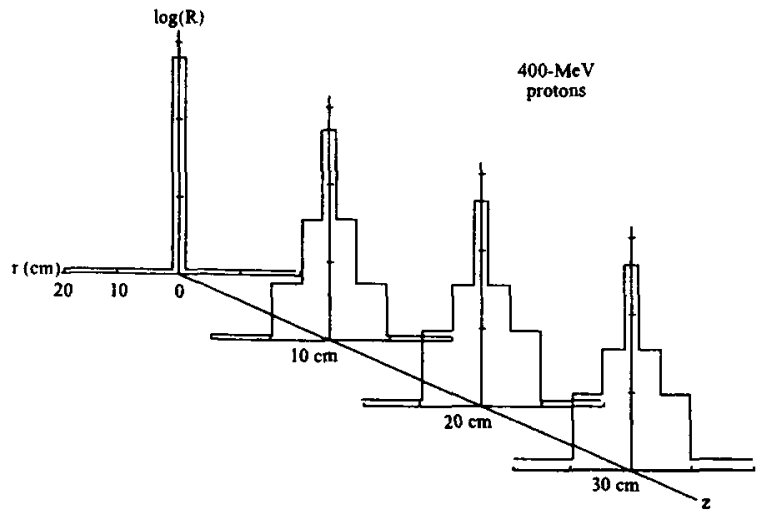

Fig. 2. Profile of $400 \mathrm{MeV}$ protons.

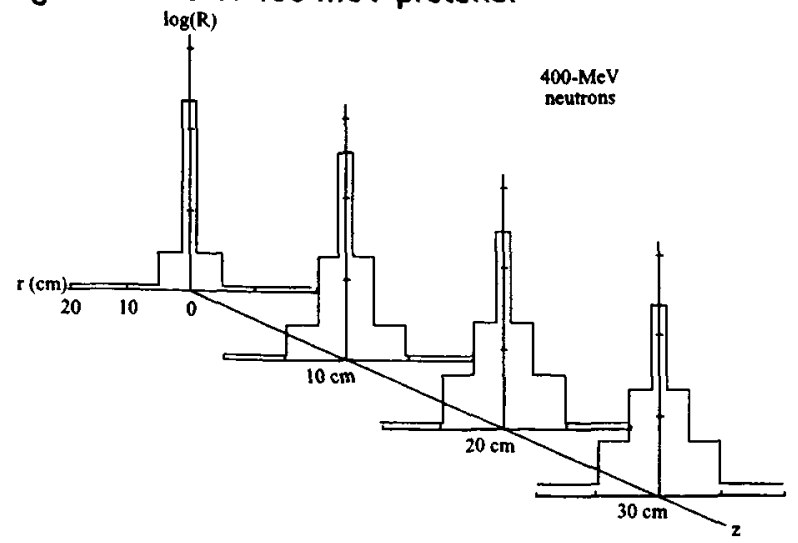

Fig. 3. Profile of $400 \mathrm{MeV}$ neutrons.

\section{PRELIMINARY CONSIDERATIONS}

Early Monte Carlo studies revealed solutions to nucleon transport that showed dose to be confined near the particle path as shown in fig. 1. The placement of near boundaries leads to leakage but is ineffective except near the central region as for boundary B. Further studies using an earty version of HETC [12] reveals similar results for high-energy proton and neutron beams as seen in figs. 2 and 3 . That even high-energy neutron beams are confined to a few centimeters of the beam axis results from the dominance of low-momentum transfer charge-exchange processes. Outside the few centimeters, the dose drops by an order of magnitude resulting from the diffuse low-energy neutron component.

Other testing with HETC for broad beams of normal and isotropic incidence protons showed only few percent differences between a straightahead approximation and full 3D calculations $[11,13]$ and provided a starting point for development of high-speed computational methods provided in part by the rapid convergence of the Neuman series for proton transport that tends to be dominated by atomic interactions at medium energies [14]. The neutron/Neuman series converges more slowly due to the neutron charge neutrality [14]. One could show that 1D errors committed in 3D-space applications was on the order of the second power of the ratio of beam divergence to the radius of curvature of space shields [11] which produces little error for human rated systems.

Adding 3D multiple-elastic scattering to the Neuman series allowed good comparisons with time-of-flight experiments with $670 \mathrm{~A} \mathrm{MeV} \mathrm{Ne}$ beams where lateral diffusion dominated the measurements (few percent detector acceptance [15,16]. Further laboratory validation with broad $\mathrm{C}, \mathrm{N}, \mathrm{O}$, and $\mathrm{Fe}$ ion beams has been completed giving confidence to our understanding of ion interactions in bulk materials [7,16-23]. The first-order Neuman series provided the basis for a high-speed marching procedure for coupled proton/neutron/HZE transport (HZETRN) in common usage in space radiation protection [24]. Target fragment contributions are evaluated analytically within the computational procedure [24-29].

Only the lateral scattering at the fragmentation event and the lateral diffusion of light ions and especially neutrons remains to be evaluated. Several approaches have been followed for a full 3D treatment of coupled neutron transport, a standard Sn code with a multigroup cross section set from reactor applications using first order finite difference methods was employed by coupling to HZETRN [30] and a separate line of development first examining mean-value multigroup methods without finite differencing and methods of collocation [31]. The HZETRN/neutron multigroup code has had reasonable success in shuttle flight validation [8]. A parallel version 
of HZETRN which operating on a 10 processor Alpha chip cluster is able to solve the Boltzmann equation for the HAB module crew quarter augmentation in few 10's of minutes which is sufficient for implementation of optimization procedures now being developed [6].

At this juncture, the deterministic evaluation of multipleelastic scattering of heavy ions has been adequately resolved with detailed laboratory testing $[15,16,32]$. The perplexity of straggling has been resolved [33]. The 3D geometry in charged particle transport can be accurately approximated by added asymptotic terms [11,34]. The lowest order asymptotic term have been solved as a marching procedure [24], a perturbation expansion [18], and a nonperturbative expansion [35], with extensive testing in laboratory and flight experiments. Detailed investigations of the diffusive neutron component at various levels of approximation from $3 \mathrm{D} \mathrm{Sn}$ methods to collocation methods including PN methods have shown promise in approaching an efficient full $3 \mathrm{D}$ code but needs further development. It has been demonstrated that these methods can be used efficiently in the full 3D complexity of spacecraft design and have been flighttested. Added treatment of the charged particle diffusive components, which will converge more rapidly than the neutral neutrons can be adequately treated using perturbation theory. A meson database needs completion [36] with addition of a complete E\&M cascade code [37]. Further considerations of fast computational procedures and implementation issues on high-performance computers for ultimate insertion into shield optimization and reliability design methods needs to be made.

\section{D CODE DEVELOPMENT}

The relevant transport equations are the linear Boltzmann equations for the flux density $\phi_{j}(x, \Omega, E)$ for particle type $j$ as

$$
\begin{aligned}
\Omega \cdot \nabla \phi_{\mathrm{j}}(\mathrm{x}, \Omega, \mathrm{E})= & \sum \int \sigma_{\mathrm{j}}\left(\Omega, \Omega^{\prime}, \mathrm{E}, \mathrm{E}^{\prime}\right) \phi_{\mathrm{k}}\left(\mathrm{x}, \Omega^{\prime}, \mathrm{E}^{\prime}\right) \mathrm{d} \Omega^{\prime} \mathrm{dE} \\
& -\sigma_{\mathrm{j}}(\mathrm{E}) \phi_{\mathrm{j}}(\mathrm{x}, \Omega, \mathrm{E})
\end{aligned}
$$

where $\sigma^{\prime}(E)$ and $\sigma_{j k}\left(\Omega, \Omega^{\prime}, E^{\prime}, E^{\prime}\right)$ are the shield media macroscopic cross sections. The $\sigma_{j k}\left(\Omega, \Omega^{\prime}, \mathrm{E}, \mathrm{E}^{\prime}\right)$ represent all those processes by which type $k$ particles moving in direction $\Omega^{\prime}$ with energy $E^{\prime}$ produce a type $\mathrm{j}$ particle in direction $\Omega$ with energy $E$ (including decay processes). Note that there may be several reactions that produce a particular product, and the appropriate cross sections for equation (1) are the inclusive ones. Exclusive processes are functions of the particle fields and may be included once the particle fields are known. The total cross section $g(E)$ with the medium for each particle type is

$$
\sigma_{j}(E)=\sigma_{,} \text {at }(E)+\sigma_{, e l}(E)+g_{, r}(E)
$$

where the first term refers to collision with atomic electrons, the second term is for elastic nuclear scattering, and the third term describes nuclear reactions where we have ignored the minor nuclear excitation processes. The corresponding differential cross section is similarty ordered. Many atomic collisions $\left(\sim 10^{6}\right)$ occur in a centimeter of ordinary matter, whereas $\sim 10^{3}$ nuclear coulomb elastic collisions occur per centimeter, while nuclear reactions are separated by a fraction to many centimeters depending on energy and particle type. Solutions use the atomic collisions as first perturbation with special methods used for neutrons for which atomic cross-sections are taken as zero.

The cross sections appearing in the Boltzmann equation are the inclusive ones so that the time-independent fields contain no spatial (or time) correlations and are evaluated once the fields are known [24]. Such correlations are important to the biological injury of living tissues [38] and transients in devices [27]. For example, the correlated release of electrons or target fragments about the ion's path determines the biological response to these ions $[26,28,38]$ and is the driver of shield material attenuation properties [39]. The solution of equation (1) involves hundreds of multi-dimensional integro-differential equations that are coupled together by thousands of cross terms and must be solved selfconsistently subject to boundary conditions.

The first order physical perturbation to the right side of equation (1) is the atomic/molecular cross sections as noted in equation (2) for which those terms in (1) are expanded about the energy moments resulting in range/energy relations including relativistic polarization effects [40] and straggling parameters $[21,24,33]$.

The distribution of the electrons about the ion path is critical to evaluation of biological injury [38], is critical to the evaluation of shield attenuation properties [39], and fundamental to dosimetric evaluation of astronaut exposure risks [41]. Such effects are likewise governed by equation (1). The next physical perturbation term is the coulomb scattering by the atomic nucleus and is represented by Rutherford scattering modified by screening of the nuclear charge by the orbital electrons using the Thomas-Fermi distribution for the atomic orbitals. The total nuclear coulomb cross section found by integrating over the scattering directions is related to the radiation length [32] and has been well validated in laboratory tests $[15,16,32]$. The electron production in ion collisions and the electromagnetic cascade following the neutral meson decay are described, in part, elsewhere $[36,37]$.

The nuclear reactive cross sections can be written in the following form

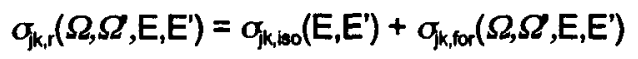


where the first term is isotropic and associated with lower energy particles produced including target fragments and the second term is highly peaked in the forward direction and is associated mainly with direct quasi-elastic events, charge exchange, and projectile fragments. Surprisingly even nucleon-induced reactions follow this simple form and the isotropic term extends to relatively high energies (see Fig. 4). For nucleon induced reactions, the following form has been used in versions of FLUKA as follows

$\sigma_{\mathrm{k}, \mathrm{r}}\left(\Omega, \Omega, \mathrm{E}^{\prime}, \mathrm{E}^{\prime}\right)=v_{\mathrm{jk}}\left(\mathrm{E}^{\prime}\right) \sigma_{\mathrm{jkr}}\left(\mathrm{E}^{\prime}\right) \mathrm{f}_{\mathrm{jk}}\left(\mathrm{E}, \mathrm{E}^{\prime}\right) \mathrm{g}_{\mathrm{R}}\left(\Omega \cdot \Omega, E, A_{\mathrm{T}}\right)$

where the Ranft factor used in FLUKA is

$$
\mathrm{g}_{\mathrm{R}}\left(\Omega \cdot \Omega^{\prime}, E, A_{\mathrm{T}}\right)=N_{\mathrm{R}} \exp \left[-\theta^{2} / \lambda_{\mathrm{R}}\right] \pi 2 \geq \theta \approx 0
$$

and constant for larger values of production angle $\theta$ and $\lambda_{R}$ given by Ranft as

$$
\lambda_{R}=\left(0.12+0.00036 A_{T} / E\right)
$$

although new generalized fits are being derived. This separation in phase space is exploited for efficient computational procedures. The heavy-ion projectile fragment cross-sections are further represented by

$$
\begin{aligned}
& \sigma_{\mathrm{j}, \mathrm{for}}\left(\Omega, \Omega^{\prime}, \mathrm{E}, \mathrm{E}^{\prime}\right)=\sigma_{\mathrm{jk}, \mathrm{r}}\left(\mathrm{E}^{\prime}\right) \mathrm{N}_{\mathrm{t}} \exp \left[-2 \mathrm{~m} \sqrt{ }\left(\mathrm{EE}^{\prime}\right)\left(1-\Omega \cdot \Omega^{\prime}\right) / \varepsilon_{\mathrm{t}, \mathrm{k} k}\right] \\
& x \exp \left[-\left(E+\lambda_{k}-E^{\prime}\right)^{2} / 2 \varepsilon_{j k}{ }^{2}\right] \sqrt{ }\left(2 \pi \varepsilon_{i k}{ }^{2}\right)
\end{aligned}
$$

where $\lambda_{j \mathrm{k}}$ is related to the momentum downshift, $\varepsilon_{\mathrm{jk}}$ is related to the longitudinal momentum width, $\varepsilon_{i, j k}$ is related to the transverse momentum width, and $N_{t}$ is the transverse normalizing factor. Since the transverse width is small compared to the projectile and fragment energy the transverse function is highly peaked about the forward direction (ie, $\Omega \cdot \Omega^{\prime}-1$ ).

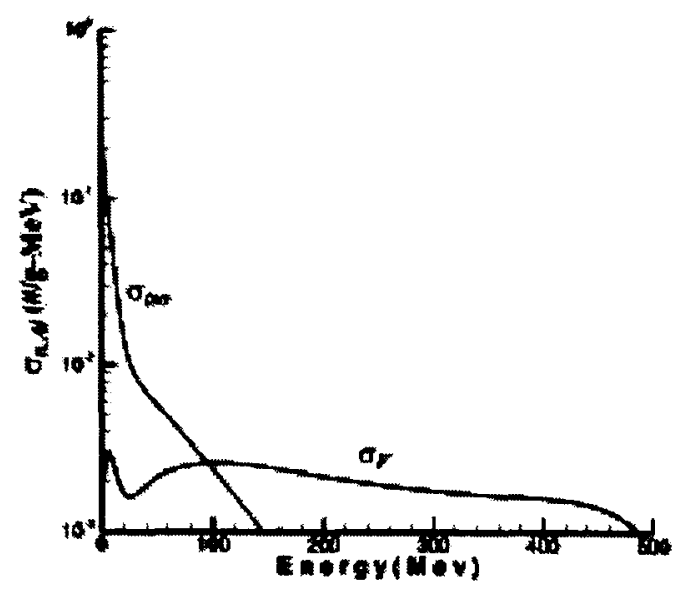

Fig. 4. Isotropic and forward neutron spectra produced bv $500 \mathrm{MeV}$ proton in aluminum.
Atomic interactions limit the contributions of charged particles in the transport process. For example, the protons and alpha particles produced in aluminum below $100 \mathrm{~A} \mathrm{MeV}$ contribute to the fluence only within a few centimeters of their collision source and the heavier ions are even more restricted (see Fig. 5). This is an important factor in that the transported secondary charged particle flux tends to be small at low energies and the role of additional nuclear reactions are likewise limited.

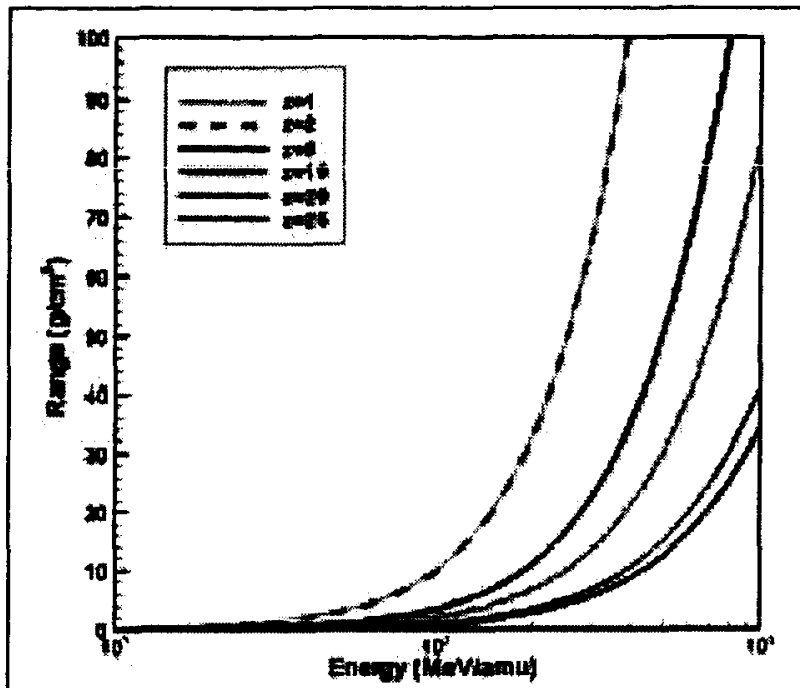

Fig. 5. Range of ions in aluminum.

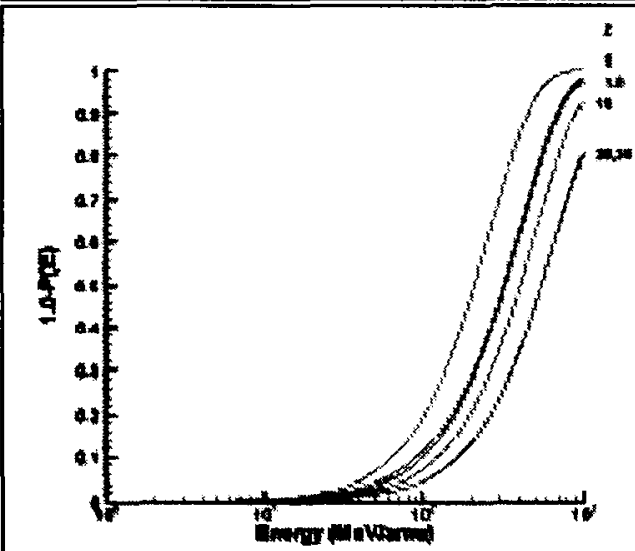

Fig. 6. Probability of nuclear reaction as a function of ion type and energy.

We rewrite equation (1) in operator notation by defining a vector array field function as

$$
\Phi=[\phi(\mathrm{X}, \Omega, \mathrm{E})]
$$

the drift operator

$$
\mathrm{D}=[\Omega \cdot \nabla]
$$

and the interaction operator

$$
I=\left[\Sigma \int \sigma_{\mathrm{jk}}\left(\Omega, \Omega, \mathrm{E}^{\prime}, \mathrm{E}^{\prime}\right) \mathrm{d} \Omega^{\prime} \mathrm{dE} \mathrm{E}^{\prime}-\sigma_{\mathrm{j}}(\mathrm{E})\right]
$$


with the understanding that I has three parts associated with atomic, elastic, and reactive processes as given in equation (2). Equation (1) is then rewritten as

$$
\left[D-I_{a t}-I_{01}\right] \cdot \Phi=I_{r} \cdot \Phi
$$

where the first two physical perturbation terms are shown on the left-hand side and have been adequately resolved in past research. The reaction cross section is separated by equation (3) into isotropic and forward component for which equation (11) may be written as coupled equations

$$
\begin{aligned}
& {\left[D-I_{\mathrm{at}}-f_{\mathrm{al}}+\sigma_{\mathrm{r}}\right] \cdot \Phi_{\text {for }}=} \\
& \left\{\sigma_{\mathrm{r}, \mathrm{for}}\left(\Omega, \Omega^{\prime}, \mathrm{E}^{\prime}, \mathrm{E}^{\prime}\right) \mathrm{d} \Omega^{\prime} \mathrm{dE}\right\} \cdot \Phi_{\text {tor }}-\Xi_{\mathrm{r}, \text { for }} \Phi_{\text {tor }}
\end{aligned}
$$

and

$$
\begin{aligned}
& {\left[D-I_{a t}-I_{a i}+\sigma_{r}\right] \cdot \Phi_{1 s 0}=\left\{\int \sigma_{r}\left(\Omega, \Omega^{\prime}, E^{\prime}, E^{\prime}\right) \mathrm{d} \Omega^{\prime} \mathrm{dE} '\right\} \cdot \Phi_{1 s 0}} \\
& +\left\{\int \sigma_{r, \text { iso }}\left(\Omega, \Omega^{\prime}, E^{\prime}, E^{\prime}\right) d \Omega^{*} \mathrm{dE}\right\} \cdot \Phi_{\text {for }} \\
& =\Xi_{\mathrm{T}} \cdot \Phi_{\mathrm{lso}}+\Xi_{\mathrm{r}, \mathrm{lsc}} \bullet \Phi_{\text {for }}
\end{aligned}
$$

Equation (12) can be solved as a Neuman series [24,34] and written as

$\Phi_{\text {for }}=\left[\mathbf{G}+\mathbf{G} \cdot \Xi_{\mathrm{r}, \text { for }} \cdot \mathbf{G}+\mathbf{G} \cdot \Xi_{\mathrm{r}, \text { for }} \cdot \mathbf{G} \cdot \Xi_{\mathrm{r}, \text { tor }} \cdot \mathbf{G}+\ldots\right] \cdot \Phi_{\mathrm{B}}$

for which the series can be either evaluated directly or proscribed as a marching procedure in either a perturbative sense as the current form of HZETRN or nonperturbative sense (future version of HZETRN) as described elsewhere [42].

The cross term in equation (13) gives rise to an isotropic source of light ions and neutrons of only modest energies for which Fig. 4 is typical. Spectral contributions to the Neuman series depend on the particle range and probability of surviving nuclear reactions that establish the functional form of the $\mathbf{G}$ matrix. The second term of the Neuman series is proportional to the probability of nuclear reaction that is limited by the particle range as discussed above and shown in Fig. 6. It is clear from Fig. 6 that nuclear reactions for the charged particles below a few hundred $\mathrm{A} \mathrm{MeV}$ are infrequent for which fast convergence has been demonstrated. For the moment we will neglect the straggling and multiple-elastic processes to simplify the present explanation (provide only minor corrections to space radiation exposures) and examine the remaining reactive terms of equation (12). The corresponding Volterra equation is given by

$$
\begin{aligned}
& \phi_{j}(\mathbf{x}, \Omega, E)=\left\{\mathrm{S}_{\mathrm{j}}\left(\mathrm{E}_{\gamma}\right) \mathrm{P}_{\mathrm{j}}\left(\mathrm{E}_{\gamma}\right) \phi_{\mathrm{j}}\left(\Gamma(\Omega, \mathbf{x}), \Omega, \mathrm{E}_{\gamma}\right)\right.
\end{aligned}
$$

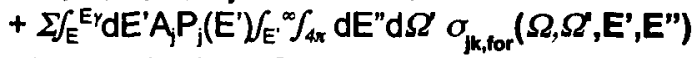

$$
\begin{aligned}
& \left.x \phi_{k}\left(x+\left[R_{j}(E)-R_{j}\left(E^{\prime}\right)\right] \Omega, \Omega^{\prime}, E^{\prime \prime}\right)\right\} / S_{j}(E) P_{j}(E)
\end{aligned}
$$

where $\Gamma$ is the point on the boundary connected to $x$ along $-\Omega, E_{Y}=R_{i}^{-1}\left[\rho-d+R_{j}\right], \rho$ is the projection of $x$ onto $\Omega$, and $d$ is the projection of $\Gamma$ onto $\Omega$. Equation (14) results from the Neuman series solution to equation (15). In the past we have expanded the angular integral $\Omega^{\prime}$ asymptotically and implemented as a marching procedure (HZETRN, [43]), as a perturbation expansion [18], and by non-perturbative approximation [44] resulting in three distinct methods to evaluate the first order asymptotic terms, all of which have had extensive experimental validation. Independent of the method used to evaluate the lowest asymptotic order term, the first correction term is found by replacing the fluence in the integrand of equation (15) by the lowest order asymptotic solution as

$$
\begin{aligned}
& \phi_{j}(X, \Omega, E)=\left\{S_{j}\left(E_{i j}\right) P_{j}\left(E_{i j}\right) \phi_{j}\left(\Gamma(\Omega, X), \Omega, E_{\gamma}\right\}\right.
\end{aligned}
$$

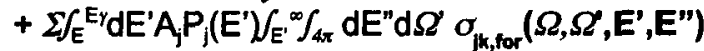

$$
\begin{aligned}
& \left.x \phi_{k, 0}\left(\mathrm{X}+\left[\mathrm{R}_{\mathrm{j}}(\mathrm{E})-\mathrm{R}_{\mathrm{j}}\left(\mathrm{E}^{\prime}\right)\right] \boldsymbol{\Omega}^{\prime}, \boldsymbol{\Omega}^{\prime}, \mathrm{E}^{\prime \prime}\right)\right\} / \mathrm{S}_{\mathrm{j}}(\mathrm{E}) \mathrm{P}_{\mathrm{j}}(\mathrm{E})
\end{aligned}
$$

where $\phi_{j}(X, \Omega, E)$ is found as an integral over the neighborhood of rays centered on $\Omega$ using the lowest order asymptotic solution $\phi_{\mathrm{k}, 0}\left(\mathrm{x}, \Omega^{\prime}, \mathrm{E}^{\prime \prime}\right)$ along an adjacent ray directed along $\Omega^{\prime}$. Note that the boundary condition reached along $-\Omega$ ' enters through the lowest order asymptotic approximation and the angular integral correction in equation (16) is determined by the homogeneity and angular dependence of the space radiation and radius of curvature of the bounding material which we have shown long ago are the determinant factors of the magnitude of the first order asymptotic correction which is anticipated to be very small for human rated systems (large radius of curvature) in space radiation which is homogeneous and isotropic in most applications $[11,24,34]$.

In a region of small radius of curvature the specific flux components near the site of evaluation will be missing contributions along adjacent rays which do not compensate losses along the ray on which the solution is evaluated representing the losses due to leakage. This computational procedure is only a small addition to prior code development and will have little impact on computational efficiency. The angular dependence of the integral kernel of equation (16) is controlled by the forward reactive cross section $\sigma_{j k, \text { tor }}\left(\Omega, \Omega^{\prime}, E^{\prime}, E^{\prime \prime}\right)$ with its highly peaked structure given by equations (4) or (7) depending on particle type. Even the most dispersed (nucleonic) components remain near the forward direction as shown in Figs. 2 and 3 . The angular dependence of the forward peak of fragmenting $\mathrm{Ca}$ ions at 100 and 1,000 A MeV is shown in Fig. 7. The lowenergy ions with limited range have transverse components on the order of 10 degrees reducing to a few degrees at high energies. Note that the low energy ions have limited range and will contribute little to the transported flux (see Fig. 5) or nuclear reactions (see Fig. 6). The higher energy ions with their much longer pathlengths giving more important contributions are related to only a very small angle of acceptance (few degrees) at the boundary. The form of the kernel leads directly to a 
- Gauss-Hermite expansion and evaluation over the angle of production. Although the neutron Neuman series for the forward components converge more slowly since their contribution to the neutron flux is not limited by atomic interactions these higher energy neutrons will be adequately evaluated by the same procedures (for example, see Fig. 3). Higher order asymptotic terms can be evaluated with similar iteration of equation (15) if required but all indications are that the first such correction will be small $[11,34]$.

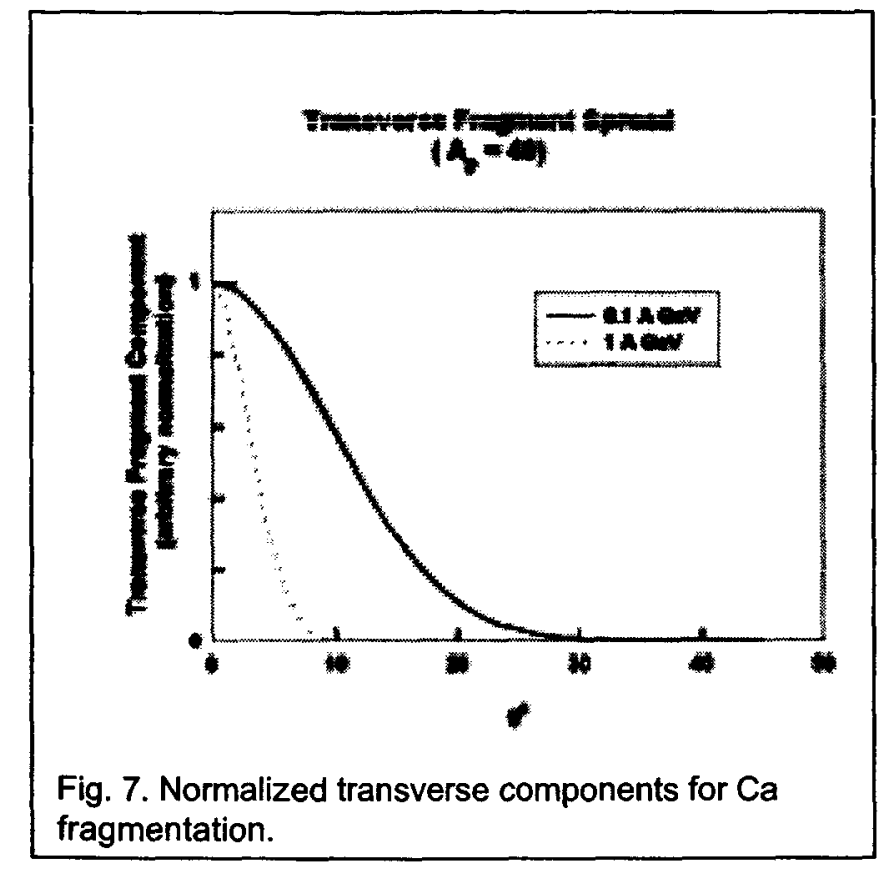

The remaining problem is solution for the transport of the low-energy neutron and light ion isotropic sources in equation (13) that dominate the solution below about 70 $\mathrm{A} \mathrm{MeV}$ (see Fig. 4). In this region light ion transport is completely dominated by the atomic interaction terms and only a very small fraction have nuclear reactions making only minor contributions to the particle fields (Fig. 6). This is especially true for the target fragments that can be solved in closed form $[25,34]$. The 3D solution for the low-energy neutrons and light ions is approached through the integral of equation (16) with the inbound flux equal to zero as

$\phi_{\mathrm{j}}(\mathrm{x}, \Omega, \mathrm{E})=$

$$
\begin{gathered}
\left\{\Sigma \int_{E} E_{\gamma} d E^{\prime} A_{j} P_{j}\left(E^{\prime}\right) \int_{E^{\prime}}{ }^{\infty} \int_{4 \pi} d E^{\prime \prime} d \Omega^{\prime} \sigma_{j k, \text { or }}\left(\Omega, \Omega^{\prime}, E^{\prime}, E^{\prime \prime}\right)\right. \\
\phi_{k}\left(x+\left[R_{j}(E)-R_{j}\left(E^{\prime}\right)\right] \Omega, \Omega^{\prime}, E^{\prime \prime}\right)+\sum \int_{E}^{E r} d E^{\prime} A_{j} P_{j}\left(E^{\prime}\right) \\
\left.\zeta_{k}\left(x+\left[R_{j}(E)-R_{j}\left(E^{\prime}\right)\right] \Omega, E^{\prime}\right)\right\} / S_{j}(E) P_{j}(E)
\end{gathered}
$$

where $\zeta_{k}\left(x, E^{\prime}\right)$ is the isotropic source from coupling to equation (13). We solve equation (17) by perturbation where the lowest order term is only the source term. We evaluate the first correction by replacing $\phi_{k}\left(x+\left[R_{j}(E)-\right.\right.$ $\left.\left.\mathrm{R}_{\mathrm{j}}\left(\mathrm{E}^{\prime}\right)\right] \Omega, \Omega^{\prime}, \mathrm{E}^{\prime \prime}\right)$ under the integral by the lowest order solution. The correction is proportional to the nuclear reaction probability as given in Fig. 6 and is quite small except for neutrons.

The neutrons have no charge and are dominated at low energies by elastic and reactive nuclear processes. We further expand equation (13) for the single neutron component as

$$
\begin{array}{r}
{\left[\Omega \cdot \nabla+\sigma_{\mathrm{n}}\right] \phi_{\mathrm{n}}(\mathrm{x}, \Omega, \mathrm{E})=\int \sigma_{\mathrm{nn}}\left(\Omega, \Omega^{\prime}, \mathrm{E}^{\mathrm{E}}, \mathrm{E}^{\prime}\right) \phi_{\mathrm{n}}\left(\mathrm{x}, \Omega^{\prime}, \mathrm{E}^{\prime}\right)} \\
\mathrm{d} \Omega \mathrm{dE}^{\prime}+\left[\Xi_{\mathrm{r}, 100} \cdot \Phi_{\mathrm{tor}}+\Xi_{\mathrm{P}} \cdot \Phi_{(14)}\right]_{\mathrm{n}}(18)
\end{array}
$$

where the last term is from coupling to the solution of equation (17) and the spectral properties are characterized by

$$
f_{1}(E)=\int f\left(E, E^{\prime}\right) f_{i s o}\left(E^{\prime}, 500 \mathrm{MeV}\right) d E^{\prime}
$$

with results shown in Fig. 8. The neutron spectrum is greatly degraded in energy on the first collision (Fig. 8) and the remaining development of the low-energy neutron transport is the last issue to be resolved. This is the typical nuclear engineering problem for which a multitude of methods have been developed such as the $\mathrm{Sn}$, multigroup, and collocation methods already applied to HZETRN. It is mainly a question of computational efficiency and we continue to investigate this issue.

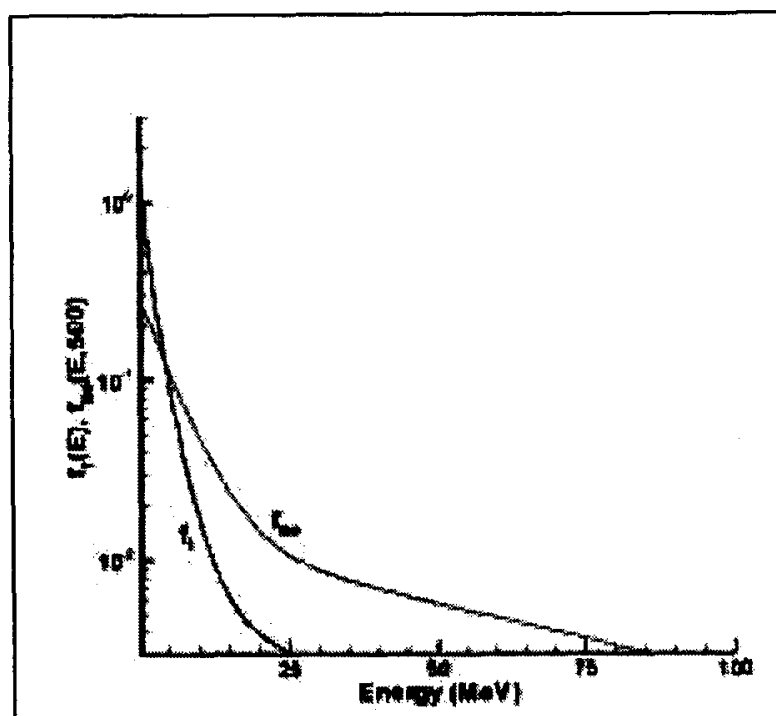

Figure. 8. Second collision neutron spectrum f1(E).

Although, equation (18) exhibits behavior similar to thermal diffusion there are strong differences between thermal and neutron diffusive processes. Thermal diffusion at ordinary temperatures has minor leakage through near boundaries since radiative processes are proportional to $\mathrm{T}^{4}$ (in the absence of convection) leaving lateral diffusion an important process. In distinction, neutron diffusion is dominated by leakage at near forward and backward boundaries in penetrating spacecraft walls and lateral diffusion play a minor role. Generally, low-energy neutron leakage is a dominant process within $15-20 \mathrm{~g} / \mathrm{cm}^{2}$ of the bounding surface in 
- most materials. Since human rated systems have shielding of large radius of curvature and small thickness to radius ratio as determined by living and working space requirements, it approximates a connected system of flat plates for which leakage at forward and backward

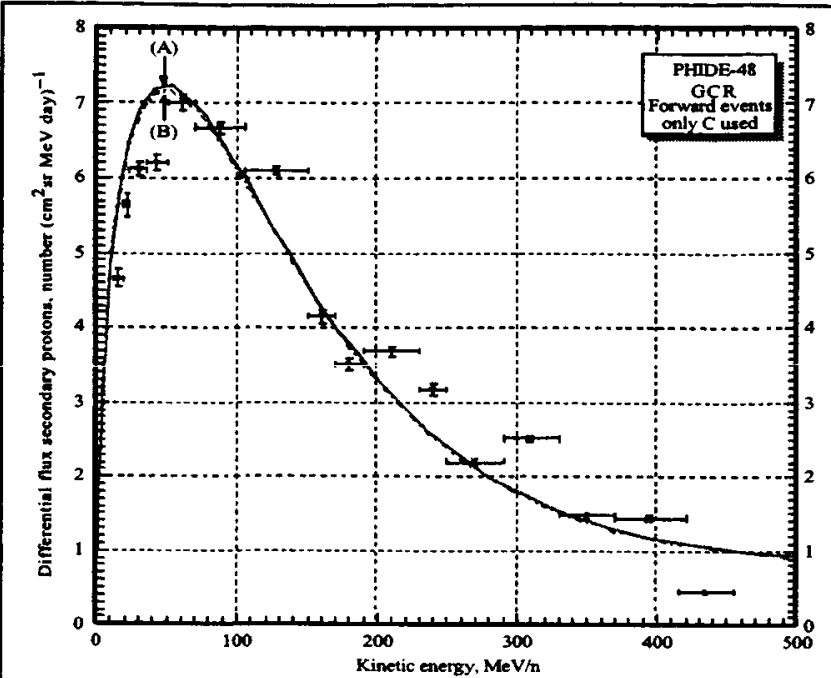

Fig. 8. Observed and calculated proton spectra.

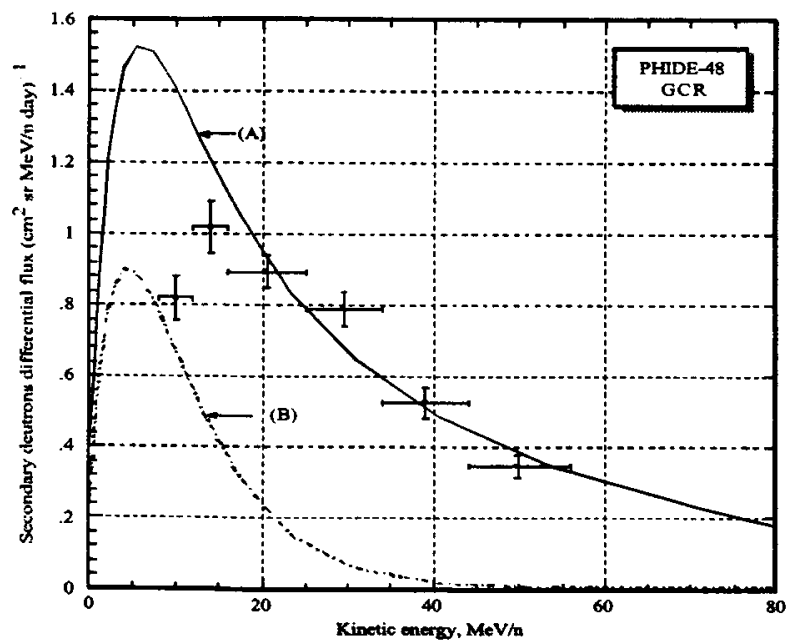

Fig. 8. Observed and calculated deuteron spectra.

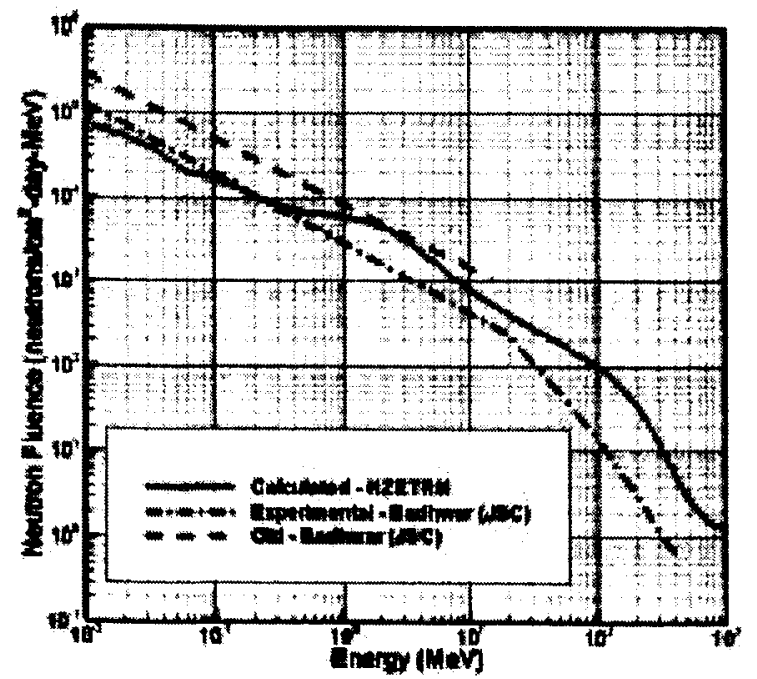

Fig 10. Observed and calculated neutron spectra. boundaries dominates the transport. In this limit the transport simplifies to a connected set of 1D transport problems with leakage at the back and forward boundaries [ 8,31$]$ and shows reasonable success in comparison with experimental flight data, see Figs. 8-10 $[45,46]$. In the present development we considered a convergent series of approximations to gauge accuracy of the transport procedures and allow choices of the most practical method in a given application. The results shown in Figs. 8-10 are the lowest order asymptotic term and the good comparison with flight data encourages us to believe that the earlier analysis on the rates of convergence of the asymptotic series $[11,34,35]$ is correct and the next corrections will be small with the possible exception of improved low energy neutron transport results. We will of course require improved neutron measurements as seen from the two analyses of the JSC bonner sphere data.

\section{CONCLUSION}

Considerable research on the development of highperformance computational procedures has been committed over the last thity years. The problem of developing a full 3D version of these procedures is now very clear and the steps required have been fully demonstrated. Even a partial solution of the problem has met with considerable success in comparison with laboratory and flight measurements. A program for developing the final software is now under way and should be complete over the next several years.

\section{REFERENCES}

1. R.G. Alsmiller, Nucl. Sci. \& Eng. 27: 158-189; 1967.

2. J.J.Lambiotte, J.W.Wilson, T.A. Filippas, Proper-3C: A nucleon-pion transport code. NASA TM X2158, 1971.

3. R.G. Alsmiller et al., Shielding of Manned Space Vehicles Against Protons and Alpha Particles. ORNL-RSIC-35, 1972.

4. T.W. Armstrong, B.L. Colburn, Radiat. Meas. 33: 229-234; 2001.

5. Aerospace Safety Advisory Panel, www.hq.nasa.gov/office/coded/codeg-1.htm, 2000.

6. G.D. Qualls et al. SAE 2001-01-2370; 2001.

7. J.W. Wilson et al., Acta Astronautica 49:289-312; 2001.

8. M.S. Clowdsley et al., SAE 2001-01-2327; 2001.

9. J.W. Wilson et al. Health Phys. 69: 34-45; 1995.

10. G.D.Qualls, R. Boykin, NASA CP-3360, pp. 365-382, 1997.

11. J.W. Wilson, G.S. Khandelwal, Nucl. Tech. 23: 298305; 1974.

12. H.A. Wright et al., International Congress on Protection Against Accelerator and Space Radiation. J. Baarli and J. Dutrannois, eds., CERN 71-16, vol. 1, pp. 207-219, 1971. 
13. R.G. Alsmiller et al., Second Symposium on Protection Against Radiations in Space. A. Reetz, ed., NASA SP-71: 177-181, 1965.

14. J.W. Wilson, S.L. Lamkin, Nucl. Sci. and Eng. 57: 292-299; 1975.

15. M.R. Shavers et al., Radiat. Res. 124: 117-130; 1990.

16. M.R.Shavers et al., Radiat. Res. 134: 1-14; 1993.

17. J.L. Shinn et al. Radiat. Meas. 23: 57-64; 1994.

18. J.W.Wilson et al., Health Phys. 46: 1101-1111; 1984.

19. S.Y. Chun et al., Nucl. Sci. \& Eng. 122: 267-275; 1996.

20. J.W. Wilson et al., Adv. Space Res. 21(12): 1763$177 \mathrm{i} ; 199 \overline{8}$.

21. J.W. Wilson, H. Tai, Range and energy straggling in ion beam transport. NASA/TP-2000-209864, 2000.

22. A.S. Golovchenko et al., Nucl. Inst. Meth. B 159:233-240; 1999.

23. J.W. Wilson et al., SAE 2001-01-2372; 2001.

24. J.W.Wilson et al., Transport Methods and Interactions for Space Radiations. NASA RP-1257, 1991.

25. F.A.Cucinotta et al. Radiat. Res. 127:130-137; 1991.

26. J.L. Shinn et al., Health Physics, 61:415-419; 1991.

27. J.L. Shinn et al., IEEE Trans. Nucl. Science, 42: 2017-2025; 1995.

28. F.A. Cucinotta et al., Radiat. Meas., 26: 923-934; 1996.

29. J.L. Shinn et al., IEEE Transactions on Nuclear Science, 45: 2711-2719; 1998.

30. S.C. Singleterry, J.W. Wilson, Angular Neutron Transport Investigation in the HZETRN Free-space Ion and Nucleon Transport and Shielding Computer Program, Advancements and Applications in Radiation Protection \& Shielding, Nashville, TN, April 19-23, 1998.
31. M.S. Clowdsley et al., Can. J. Phys. 78: 45-56; 2000.

32. W. Schimmerling et al. Med. Phys. 13: 217-228; 1986.

33. J.W. Wilson et al., Nucl. Inst. \& Meth. TBP.

34. J.W. Wilson, Analysis of the theory of high-energy ion transport. NASA TN D-8381, 1977.

35. J.W.Wilson et al., Adv. Sp. Res. 14(10): 841$852 ; 1994$.

36. F.A. Cucinotta et al. Parameterization of Pion Energy Spectrum in Nucleon-Nucieon Collisions, NASA Technical Memorandum 1998-208722, 1998.

37. J.E.Nealy et al. Transport of space environment electrons: A simplified rapid analysis computational proceedure. NASA/TP-2002-211448, 2002.

38. F.A. Cucinotta et al., Int. J. Radiat. Biol. 69:593-600; 1995.

39. J.W. Wilson et al. , Health Phys. 68:50-58; 1995.

40. J.L. Shinn et al. Polarization Correction for lonizaion Loss in a Galactic Cosmic Ray Transport Code (HZETRN). NASA TM 4443, 1993.

41. J.L. Shinn et al. Health Physics 76: 510-515; 1999.

42. J.W. Wilson et al. Radiat. Res. 140: 241-244; 1994.

43. J.W. Wilson et al. HZETRN: Description of a FreeSpace Transport and Shielding Program. NASA TP-3495, 1995.

44. J.W.Wilson et al. Radiat. Res. 140: 241-244; 1994.

45. G.D. Badhwar et al. Radiat. Meas. 24:129-138; 1995.

46. G.D. Badhwar et al. Radiat. Meas. 33:235-241; 2001.

\section{CONTACT}

John.w.wilson@larc.nasa.gov 